\title{
Newly dentified members of the TNF receptor superfamily (mTNFRH1 and mTNFRH2) inhibit T-cell proliferation
}

\author{
Juyang Kim, Soojin La', Byung-Sam Kim, \\ Byoung Se Kwon ${ }^{1}$ and Byungsuk Kwon ${ }^{1,2}$ \\ ${ }^{1}$ The Immunomodulation Research Center
University of Ulsan, Ulsan 680-749, Korea
${ }^{2}$ Corresponding author: Tel, 82-52-259-2860;
Fax, 82-52-259-2740; E-mail, bkwon@mail.ulsan.ac.kr
}

Accepted 1 April 2003

Abbreviations: APC, antigen-presenting cell; $C R D$, cysteine-rich domain; CTL, cytotoxic T cell; TCR, T-cell receptor

\begin{abstract}
By searching an EST database, we identified two TNF receptor superfamily members (named mTNFRH1 and mTNFRH2). Amino acid sequences are highly conserved between the two receptors (78\% identity). The chromosomal loci of $m$ Tnfrh1 and $m$ Tnfrh 2 genes are found in distal chromosome 7 in the mouse. mTNFRH1 and mTNFRH2 do not contain the cytoplasmic domain, indicating that they might function as decoy receptors. Furthermore, an alternatively spliced form of mTNFRH1 was found which contains neither the transmembrane domain nor the cytoplasmic domain, thus presumably existing as a soluble form. Northern blot analysis showed that mTnfrh1 mRNA was negligibly expressed in tissues, while mTnfrh2 mRNA was strongly expressed in spleen, lung, liver, kidney, and testis. When the extracellular domains of mTNFRH1 and mTNFRH2 were expressed in bacteria, their molecular weight of extracellular region was approximately $15 \mathrm{kDa}$. Both of the soluble forms were effective in inhibiting T-cell proliferation stimulated by anti-CD3 monoclonal antibody. Our data suggest that mTNFRH1 and mTNFRH2 may be implicated in exerting a modulatory role in the immune response.
\end{abstract}

Keywords: decoy receptor; T-cell proliferation; TNF receptor superfamily

\section{Introduction}

TNF receptor superfamily members have common motifs containing cysteine-rich pseudorepeats in the extracellular domain (Locksley et al., 2001; Kwon et al., 2003). In contrast to the extracellular domain, the cytoplasmic domain does not share any distinctive motifs or even any significant sequence homology, except that of the death domain-containing members such as Fas, TNFR1, DR3, DR4, DR5, and DR6. At present, more than 20 TNF receptor family members have been identified. Molecules of the TNF receptor and ligand superfamilies play a central role in regulating immune responses, ranging from cell survival to apoptosis. However, their functions are far more diverse, extending beyond the immune responses, including embryonic development, organogenesis, and metabolism.

Recently, several research groups have identified decoy receptors (DcRs) that can't deliver a signal into cells because of absence of the cytoplasmic domain or existence of its incomplete form. Thus, DcRs belonging to the TNF receptor family exist as either a soluble form or a membrane-bound form. DcRs identified so far include DcR1 and DcR2 for TRAIL (Delgli-Esposti et al., 1997a; Delgli-Esposti et al., 1997b; Masters et al., 1997; Pan et al., 1997; Sheridan et al., 1997), DcR3 for FasL and LIGHT (Pitti et al., 1998; Yu et al., 1999), and OPG for TRANCE (Simonet et al., 1997). DcR1 and DcR2 are preferentially expressed in tumor cells, being implicated in immune evasion mechanism for tumor cells (for review, see Ashkenazi, 2002). Similarly, various tumor cells are thought to escape from the attack of cytotoxic $T$ cells (CTLs) by producing DcR3, which can block interaction between Fas on tumor cells and FasL on CTLs (Pitti et al., 1998). OPG is secreted by osteoblasts and is highly effective in inhibiting osteoclast differentiation and activation stimulated by signaling via RANK, the receptor for TRANCE (for review, see Kong et al., 2000).

Here, we report identification of two new decoy receptors with a high sequence homology to each other. Our data suggest that they may function as immunomodulatory molecules.

\section{Materials and Methods}

Identification and cloning of $m$ Tnfrh1 and mTnfrh2 cDNAs

The cDNAs of mTnfrh1 and mTnfrh2 genes were identified by searching an EST database (GenBank 
accession number Al747041 and AK012838, respectively). For cloning of full-length cDNAs, total RNA was prepared from mouse spleen using Trizol $^{\mathrm{TM}}$ reagent (Life Technologies, Gaithersburg, MD). cDNA was prepared by reverse transcription of total RNA using the Superscript Preamplification System (Life Technologies, Rockville, MD). A full-length cDNA was amplified by polymerase chain reaction (PCR) using sense (5'- TGCTAGCTCACAGCCATG-3' for mTnfrh1; 5'-TCGGCCATGTTTGGCTTC-3' for mTnfrh2) and antisense (5'-AGCAATAAAAGGAAGCTA-3' for mTnfrh1; 5'-GTGAACCATTCAGATAGG-3' for $m$ Tnfrh2) primers, and inserted into PGEM-T easy vector (Promega, Madison, WI).

\section{Northern blot analysis}

For Northern blot analysis, cDNA probes were labeled with $\alpha^{32}$-dCTP using Ready-to-Go DNA Labeling Beads (Amersham Pharmacia Biotech, Piscataway, $\mathrm{NJ}$ ), according to the manufacturer's instructions. Unincorporated nucleotides were removed from the labeled probe using CHROMA SPIN-100 (CLONTECH Laboratories, Palo Alto, CA). A mouse multiple tissue poly (A) RNA blot containing approximately $2 \mu \mathrm{g}$ of poly (A) RNA per lane from various mouse tissues was purchased from CLONTECH. Northern blotting was performed with the Expressed Hybridization Solution (CLONTECH) according to the manufacturer's instruction.

\section{Production of recombinant proteins in Escherichia coli}

Histidine-tagged mTNFRH1 and mTNFRH2 encoding the putative extracellular domain were amplified by PCR using sense (5'-CTAGTCTAGACATGCCTGAATCATACTCC-3' for mTnfrh1; 5'-CTAGTCTAGACATGCTAGAATTACACTCC-3' for $m$ Tnfrh2) and antisense (5'-CCGCTCGAGTCAGTGGTGGTGGTGGTGGTGGT GCACAGTGTTAGCTGTGGA-3' for mTnfrh1; 5'-CCGCTCGAGTCAGTGGTGGTGGTGGTGGTGGTGCACA GTGGCACACAGTGTTAGCTGT-3' for $m$ Tnfrh2) primers, cut with $X b a l / X h o l$, and fused in frame upstream of a glutathionine S-transferase (GST) sequence into pGEX-3 (Amersham Pharmacia, Uppsale, Sweden). Each of the in-frame fusions was confirmed by sequencing. The pGEX-3-transformed $E$. coli were then grown to an optical density of 0.6 (A590) before protein expression was induced by the addition of $2 \mathrm{mM}$ isopropylthiogalactopyranoside (IPTG). The mTNFRH1- and mTNFRH2-GST fusion proteins were purified by two-step chromatography with GST-Sepharose $4 \mathrm{~B}$ and Ni-column (Novagen, Madison, WI). The column eluents were fractionated by SDS-PAGE, and the recombinant proteins were detected by
Western blot using the anti-poly (His) monoclonal antibody (R\&D Systems, Minneapolis, MN).

\section{T-cell proliferation assay}

Spleens were isolated from C57BL/6 mice and a single cell suspension was obtained in PBS. The cell debris was removed by passing through $70-\mu \mathrm{m}$ cell strainer (BD Falcon, San Diego, CA). The red blood cells (RBCs) were lysed using ACK lysis buffer (BioSource International, Camarillo, CA). Splenocytes $\left(5 \times 10^{5}\right)$ were cultured in the presence of $1 \mu \mathrm{g}$ of soluble anti-CD3 monoclonal antibody, $5 \mu \mathrm{g} / \mathrm{ml}$ of polymyxin (Sigma, St. Louis, MO), and various concentrations of recombinant mTNFRH1-GST, mTNFRH2GST, or GST for 48 h. Proliferation was assessed in triplicate by pulsing with $1 \mu \mathrm{Ci}\left[{ }^{3} \mathrm{H}\right]$ thymidine for 18 h.

\section{Results \\ Identification of murine Tnfrh1, Tnfrh2 and their variants}

The hallmark of the TNF receptor superfamily is the existence of cysteine-rich domains (CRDs) that contain approximately 40 amino acids. These CRDs are typically defined by three disulfide bonds generated by six highly conserved cysteines. A specific homology and motif search using the first CRD of TNFR1 against an NIH EST database revealed several ESTs clones. We cloned the full-length cDNA from mouse spleens using RT-PCR. A full-length $m$ Tnfrh1 cDNA encodes 176 amino acid type I transmembrane protein (Figure $1 \mathrm{~A}$ ). The receptor has a signal peptide and a single short transmembrane domain but no cytoplasmic domain. When compared with the extracellular domains of other TNF receptor family members, mTNFRH1 displays three CRDs. The first CRD is the prototype of CRDs corresponding to that found in many TNF receptor family members, including TNFR I and TNFR II. The second and third CRDs show extensive homologies with that found in Troy, $\mathrm{TACl}, \mathrm{BCMA}$, and EDAR. All three CRDs contain six conserved cysteines, indicating the existence of the canonical pattern of $\mathrm{C} 1-\mathrm{C} 2, \mathrm{C} 3-\mathrm{C} 5$ and $\mathrm{C} 4-\mathrm{C} 6$ disulfide bonds. mTNFRH2 has 180 amino acids with a similar structure with mTNFRH1. In addition, they have high amino acid sequence homology $(78 \%$ identity).

Like other TNF receptor family members, there were alternatively spliced $m T n f r h 1$ and $m T n f r h 2$ variants (Figure 1A). Genomic sequence analysis (GenBank accession number AJ276505) revealed that the variants were indeed originated from alternative splicing (Figure 2). The mTnfrh1 cDNA spanning exons 1-6 
A

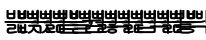

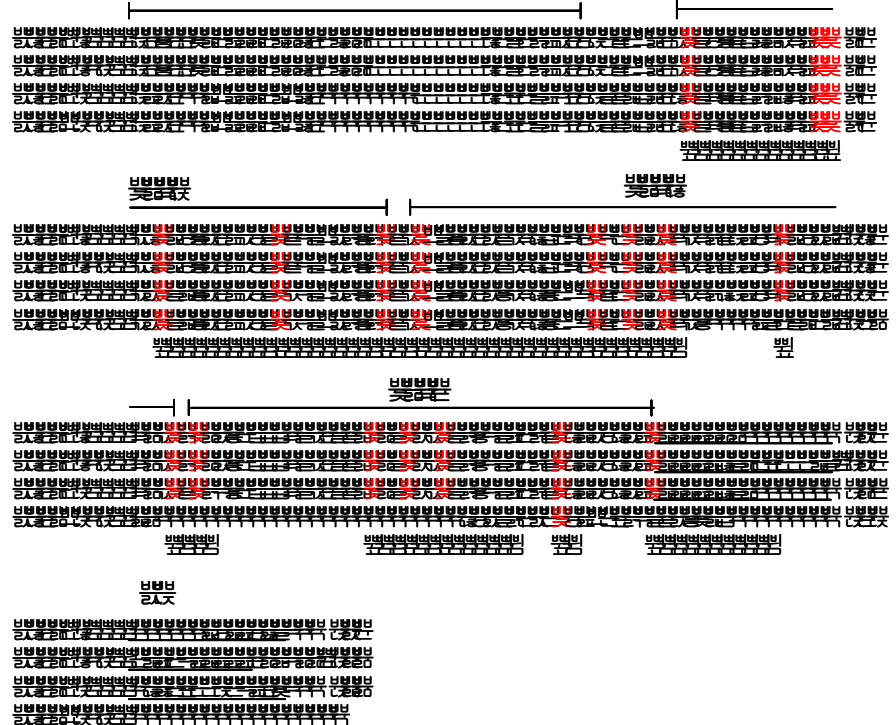

\section{B}

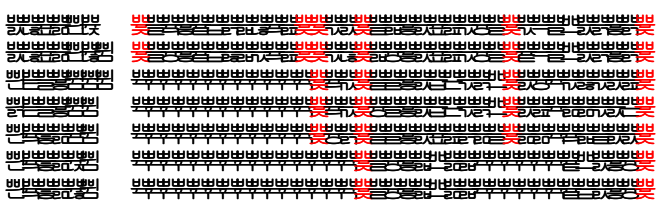

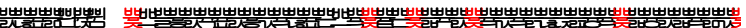

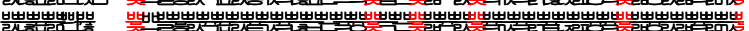

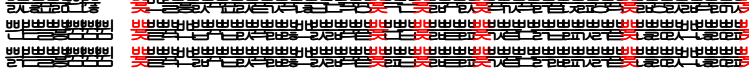

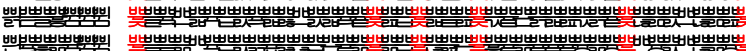

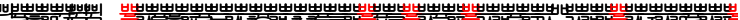

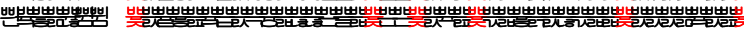

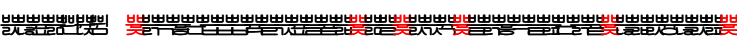

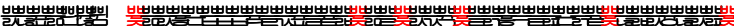

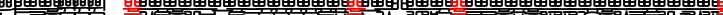

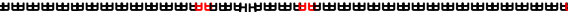

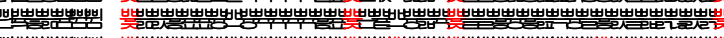

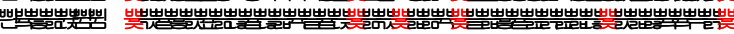

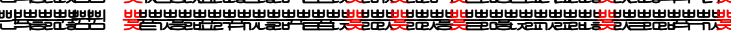

Figure 1. (A) Alignment of the deduced amino acid sequences of mTNFRH1, mTNFRH1-1, mTNFRH2, and mTNFRH2-1. A signal peptide and three cyteine-rich domains (CRDs) are indicated above the alignment. A transmembrane domain is underlined. The conserved cysteine residues within CRDs are indicated by stars under the alignment. (B) Alignment of the CRD regions of mTNFRH1/mTNFRH2 and all other known decoy receptors. Shown here are the amino acid sequences for CRDs 1-3 of the decoy receptors.

Exon

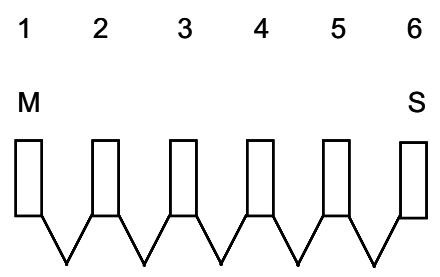

M<smiles>C1CC2CCCC(CC3CCCC4CCCC3C4)C(C1)C2</smiles>

M

S

Tnfrh2

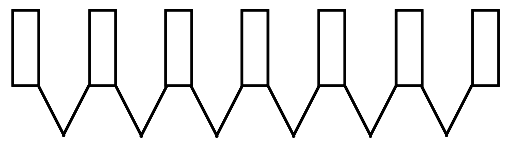

M

S

Tnfrh2-1

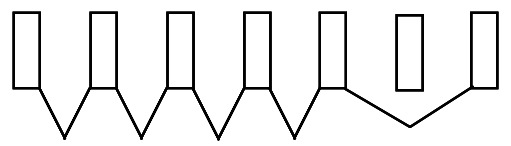

Figure 2. Genomic structure of mTnfrh1, mTnfrh1-1, mTnfrh2, and mTnfhr2-1 on mouse chromosome 7 . The mTnfrh1 gene consists of 6 exons but the mTnfrh1-1 cDNA lacks exon 4 . The mTnfrh2 gene consists of 7 exons but the stop codon is located in exon 6 . The mTnfrh2-1 cDNA encodes an alternatively spliced isoform that lacks exon 6. M: Initiation codon for Methionine; S: Stop codon. The diagram is drawn schematically, not following the original scale. contains a transmembrane region, whereas a splice variant, mTnfrh1-1 cDNA, lacking exon 4 (Figure 2), encodes a shorter secreted receptor without the transmembrane domain (Figure 1A). Since mTNFRH1-1 has virtually no third CRD, it would be interesting to examine whether the soluble mTNFRH1-1 receptor shares the same ligand with mTNFRH1. The mTnfrh2 cDNA spans exons 1-7 but it contains a stop codon within exon 6 , whereas a splice variant, $m$ Tnfrh2-1 cDNA, lacks exon 6 and encodes a longer isoform containing a transmembrane domain and a very short cytoplasmic region (Figure 2).

\section{Expression patterns of $m$ Tnfrh1 and $m$ Tnfrh2}

We investigated expression of $m T$ Trhh1 and mTnfrh2 in multiple mouse tissues by Northern blot hybridization (Figure 3). Approximately $1.5 \mathrm{~kb}$ mRNA of $m$ Tnfrh1 was weakly detected in heart and skeletal muscle. We also detected $4.0 \mathrm{~kb}$ mRNA in heart and, more weakly, in kidney. Surprisingly, high levels of $m$ Tnfrh2 mRNA were expressed in spleen, liver, kidney, testis, and lung under the same experimental conditions that were used for the examination for $m$ Tnfrh1 expression. There was heterogeneity in the transcript size among tissues. This is not uncommon in TNF receptor family members because of the existence of many alternatively spliced forms. Since we used a full-length cDNA as a probe, it is possible that any splice variants could be detectable in our ex- 

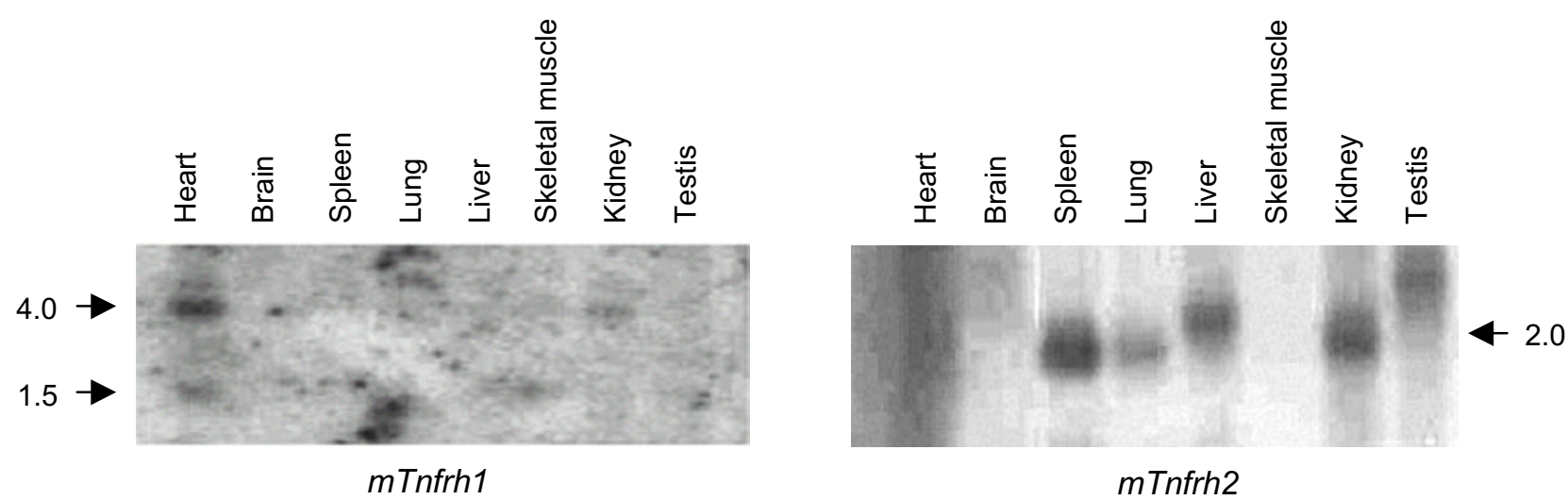

Figure 3. Expression of $m T n f r h 1$ and $m T n f h r 2$ in adult tissues by Northern blot analysis. Two mRNA species of $m T n f r h 1$ were weakly detected in tissues. Approximately 1.5-kb mRNA of $m T n f r h 2$ was detected in spleen, lung, and kidney, and larger sizes of mRNA were detected in liver and testis.

TNFRH1-GST

TNFRH2-GST
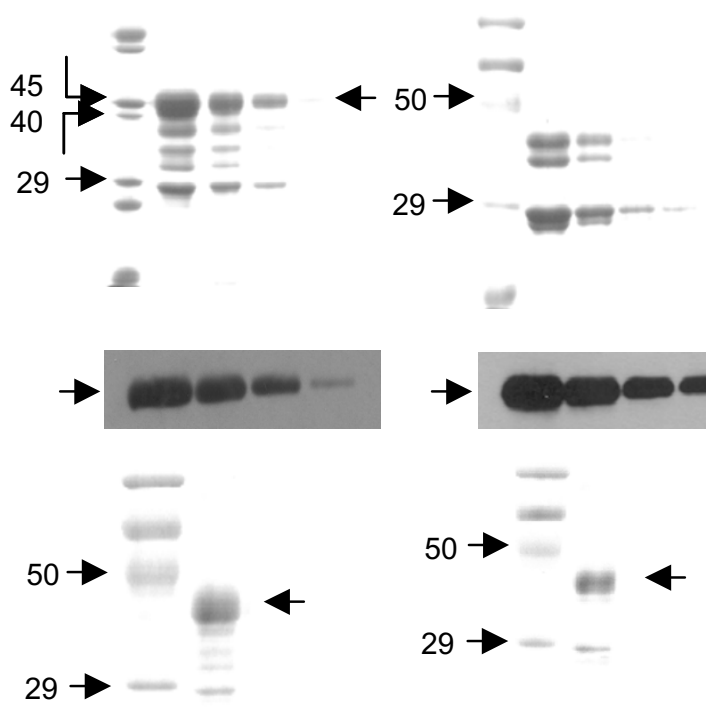

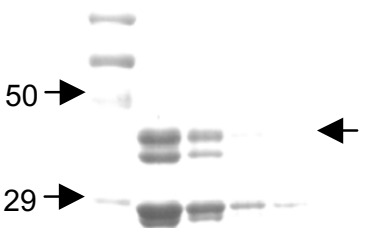

Figure 4. Production of recombinant mTNFRH1-GST and mTNFRH2GST proteins. Upper panel, recombinant proteins were purified using GST-agarose beads. Middle panel, the estimated sizes of the recombinant proteins were confirmed by Western blot analysis. The fractions shown in the upper panel were detected by anti-poly(His) monoclonal antibody. Lower panel, the fractions shown in the upper panel were pooled and further purified using Ni-column.

perimental condition. Even though mTnfrh1 expression was not detectable in spleen by Northern blot analysis, we could confirm that $m T$ frrh 1 are indeed an expressed gene in spleen by RT-PCR (In fact, we cloned the full-length $m T$ Tnfrh1cDNA from spleen). Our data indicate that despite their high homology, expression levels of $m T n f r h 1$ and $m$ Tnfrh 2 are different in mouse tissues. Our data also indicate that $m$ Tnfrh2 is a major expressed gene in many tissues and that expression of $m T$ Tnfrh 1 and $m T$ Thrh 2 might depend on tissue types.

\section{Production of recombinant mTNFRH1 and mTNFRH2 proteins}

For examination of the biological function of and production of monoclonal antibody against mTNFRH1 and mTNFRH2, we produced mTNFRH1- and mTNFRH2-GST fusion proteins (Figure 4). For the convenience of purification, we tagged seven histidine residues to the carboxyl terminus of mTNFRH1 and mTNFRH2. Soluble forms of the recombinant proteins were produced in E. coli, and purified through GSTagarose beads (Figure 4, upper panel). The expected size of mTNFRH1 and mTNFRH2 was confirmed by Western blot analysis (Figure 4, middle panel). Apporximately $15 \mathrm{kDa}$ was the size of the extracellular portion of the recombinant proteins. Higher purity of the recombinant proteins was obtained through $\mathrm{Ni}$ column (Figure 4, lower panel). These recombinant proteins were used for the experiments described below.

\section{Inhibition of T-cell proliferation by recombinant mTNFRH1 and mTNFRH2 proteins}

As described before, the mTfnrh1 and mTnfrh2 RNAs were shown to be expressed in splenocytes by RTPCR. It is possible that their respective ligand as yet unknown might be expressed in splenocytes, as seen in other TNF receptor-ligand systems. In addition, signaling via TNF receptor members costimulates $T$ cells together with the T-cell receptor (TCR) or induces apoptosis in $\mathrm{T}$ cells. We investigated the possible involvement of mTNFRH1 and mTNFRH2 in T-cell 


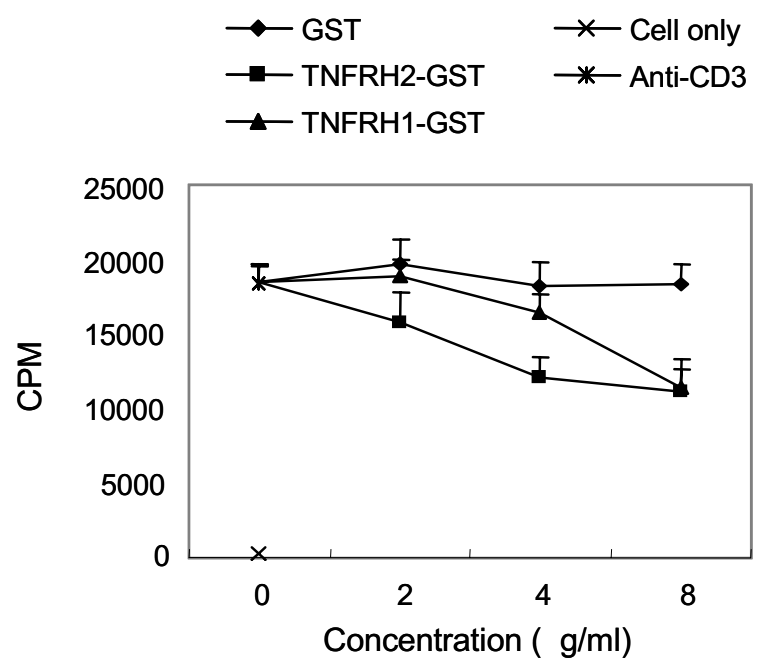

Figure 5. Inhibition of T-cell proliferation by mTNFRH1-GST or mTNFRH2-GST proteins. Splenocytes $\left(5 \times 10^{5}\right)$ were cultured in the presence of $1 \mu \mathrm{g}$ of anti-CD3 monoclonal antibody, $5 \mu \mathrm{g} / \mathrm{ml}$ of polymyxin, and various concentrations of recombinant mTNFRH1-GST, mTNFRH2-GST, or GST for $48 \mathrm{~h}$. Proliferation was assessed in triplicate by pulsing with $1 \mu \mathrm{Ci}\left[{ }^{3} \mathrm{H}\right.$ ]thymidine for $18 \mathrm{~h}$. Experiments are repeated at least three times and similar results were obtained.

proliferation. Since the ligand for mTNFRH1 or mTNFRH2 could be expressed either in T cells or in antigen-presenting cells (APCs), we used total splenocytes instead of purified T cells. Our result showed that mTNFRH2-GST recombinant proteins were able to inhibit T-cell proliferation stimulated by TCR (anti-CD3 monoclonal antibody) in a concentrationdependent manner (Figure 5). mTNFRH1-GST was less effective than mTNFRH2-GST but showed a similar efficacy at a high concentration in inhibiting T-cell proliferation. In this experiments, we used GST recombinant protein as a negative control. Recombinant GST protein could not inhibit T-cell proliferation at any points of concentration. Since the recombinant proteins were derived from bacteria, we excluded the effects of LPS by treating the culture with polymyxin, an LPS inhibitor. Our results indicate that TNFRH2 may play a more important role during the process of T-cell expansion/regression in spleen. This hypothesis is supported by the observation that $m$ Tnfrh2 mRNA was more abundant in spleen than was mTnfrh2 mRNA.

\section{Discussion}

mTnfrh1 and mTnfrh2 are located between Obph1 and Cars in the distal region of mouse chromosome 7 (Engelmann et al., 2000). Genes in this region show conserved synteny with genes on human chromosome11p15.5. Parental imprinting of genes in this chromosomal region is implicated in the human Beckwith-Wiedemann Syndrome (BWS) (Maher et al., 2000). However, it seems that mTnfrh1 and mTnfrh2 are not involved in BWS pathogenesis, as mTnfrh1 and $m T n f r h 2$ appear to be weakly imprinted in several organs and as no human orthologues of mTnfrh1 and mTnfrh2 are found in the human syntenic region (Engelmann et al., 2000; Clark et al., 2002). Clustering of the two genes, together with their high homology, indicates that they diverged by gene duplication.

The ligand for mTNFRH1 or mTNFRH2 has not been identified yet. Our binding assay showed that either mTNFRH1 or mTNFRH2 was not able to bind mouse TNF- $\alpha$ (data not shown). Since both mTNFRH1 and $\mathrm{mTNFRH} 2$ were able to inhibit T-cell proliferation induced by TCR stimulation, it is believed that both mTNFRH1 ligand and mTNFRH2 ligand are expressed in splenocytes such as T cells and APCs. In the same context, the genuine receptor for mTNFRH1 or mTNFRH2 is (are) presumably expressed in splenocytes. Sequence alignment of CRDs of mTNFRH1/ mTNFRH2 with those of other decoy receptors revealed that there are significant homologies between mTNFRH1/mTNFRH2 and TRAIL decoy receptors (Figure 1B). Even though showing a different general structure, CRDs $1-3$ of mTNFRH1/mTNFRH2 and those of other decoy receptors are highly homologous with a range of $32-36 \%$ identity at the amino acid level. Importantly, mTNFRH1/mTNFRH2 and $h D c R 1 / h D c R 2$ share the same general structure of CRDs 2-3, which provide the contact region for ligand binding (Bodmer et al., 2002). In addition, the amino acid residues in $\mathrm{hDcR} 1 / \mathrm{DcR} 2$ that interact with TRAIL are well conserved in mTNFRH $1 / m T N F R H 2$. This strongly suggests that mTNFRH1 and mTNFRH2 may function as decoy receptors for mouse TRAIL.

Due mainly to the potential clinical importance, a great deal of efforts have been invested to identify new members of the TNF receptor and ligand superfamilies by searching EST databases (Kwon and Kwon, 1999). Functional characterization of the new members identified by this approach has revealed many interesting features that were unknown previously. Some TNF family members and their ligands play an important role in other than the immune system, for example, in bone metabolism (Simonet et al., 1997) and in the entry process of virus infection (Montgomery et al., 1997). Certain TNF receptor family members function as decoy receptors. In general, these DcRs have the capacity to bind multiple ligands, implying that they are involved in a complex regulatory network in the immune system. In this study, we report the identification of two putative DcRs. Our current data indicate that these receptors may be important for modulating immune responses. Gen- 
eration of mice deficient in or overexpressing these DcRs will allow definitive analysis of their in vivo roles.

\section{Acknowledgment}

This work was supported by grants from the SRC to the IRC Fund from the KOSEF and the Korean Ministry of Science and Technology.

\section{References}

Ashkenazi A. Targetting death and decoy receptors of the tumor necrosis factor superfamily. Nat Rev Immunol 2002; 2:420-30

Bodmer JL, Schneider P, Tschopp J. The molecular architecture of the TNF superfamily. Treds Biochem Sci 2002;27: $19-26$

Clark L, Wei M, Cattoretti G, Mendelsohn C, Tycko, B. The Tnfrh1 (Tnfrsf23) gene is weakly imprinted in several organs and expressed at the trophoblast-decidua interface. BMC Genet 2002;3:11-21

Delgli-Esposti MA, Smolak $P$, Walczak $H$, Waugh $H$, Huang $\mathrm{CP}$, DuBose RF, Goodwin RG, Smith CA. Cloning and characterization of TRAIL-R3, a novel member of the emerging TRAIL receptor family. J Exp Med 1997a;186:1165-70

Delgli-Esposti MA, Dougall WC, Smolak PJ, Waugh HY, Smith CA, Goodwin RG. The novel receptor TRAIL-R4 induces NF-kB and protects against TRAIL-mediated apoptosis, yet retains an incomplete death domain. Immunity 1997b; 7:813-20

Engelmann S, Strodicke M, Paulsen M, Franck O, Reinhardt $R$, Lane N, Reik W, Walter J. Sequence and functional comparison in the Beckwith-Wiedemann region: Implications for a novel imprinting center and extended imprinting. Hum Mol Genet 2000;9:2691-760

Kong YY, Boyle WJ, Penninger JM. Osteoprotegerin ligand: A regulator of immune responses and bone physiology. Immunol Today 2000;21:495-502
Kwon B, and Kwon BS. Molecules of the tumor necrosis factor (TNF) receptor and ligand superfamilies: Endless stories. J Biochem Mol Biol 1999;32:419-28

Kwon B, Kim BS, Cho HR, Park JE, Kwon BS. Involvement of tumor necrosis factor receptor superfamily (TNFRSF) members in the pathogenesis of inflammatory diseases. Exp Mol Med 2003;35:8-16

Locksley RM, Killeen N, Lenardo MJ. The TNF and TNF receptor superfamilies: Integrating mammalian biology. Cell 2001;104:487-501

Maher ER, Reik W. Beckwith-Wiedemann syndrome: Imprinting in clusters revisited. J Clin Invest 2000;105:247-52

Masters SA, Sheridan JP, Pitti RM, Huang A, Skubatch M, Baldwin D, Yuan J, Gurney A, Goddard AD, Godowski P, Ashkenazi A. A novel receptor for Apo2L/TRAIL contains a truncated death domain. Curr Biol 1997;7:1003-6

Montgomery RI, Warner MS, Lum BJ, Spear PG. Herpes simplex virus-1 entry into cells mediated by a novel member of the TNF/NGF receptor family. Cell 1997;87:427-36

Pan G, Ni J, Wei YF, Yu GL, Gentz R, Dixit VM. An antagonsist decoy receptor and a death domain-containing receptor for TRAIL. Science 1997;277:815-8

Sheridan JP, Masters SA, Pitti RM, Gurney A, Skubatch M, Baldwin D, Ramakrishnan L, Gray CL, Baker K, Wood WI, Goddard AD, Godowski P, Ashkenazi A. Control of TRAILinduced apoptosis by a family of signaling and decoy receptors. Science 1997;277:818-21

Simonet WS, Lacey DL, Dunstan CR, Kelley M, Chang MS, Luthy $R$, Nguyen $H Q$, Wooden $S$, Bennett $L$, Boone $T$, Shimamoto G, DeRose M, Elliott R, Colombero A, Tan HL, Trail G, Sullivan J, Davy E, Bucay N, Renshaw-Gegg L, Highes TM, Hill D, Pattison W, Campbell P, Sander S, Van G, Tarpley J, Derby P, Lee R, Amgen EST Program, Boyle WJ. Osteoprotegerin: A novel secreted protein involved in the regulation of bone density. Cell 1997;89:309-19

Yu KY, Kwon B, Ni J, Zhai Y, Ebner R, Kwon BS. A newly identified member of tumor necrosis factor receptor superfamily (TR6) suppresses LIGHT-mediated apoptosis. J Biol Chem 1999;274:13733-6 